\title{
Sudden Onset of Brain Metastasis despite the Use of Vemurafenib for Another Metastatic Lesion in Malignant Melanoma Patients
}

\author{
Keisuke Imafuku ${ }^{a} \quad K^{\prime} j i$ Yoshino $^{a} \quad K^{2}$ Yamaguchi ${ }^{a}$ Satoshi Tsuboi ${ }^{a}$ \\ Kuniaki Ohara ${ }^{a}$ Hiroo Hata $^{b}$ \\ ${ }^{a}$ Tokyo Metropolitan Cancer and Infectious Diseases Center, Komagome Hospital, \\ Tokyo, Japan; ${ }^{b}$ Department of Dermatology, Hokkaido University Graduate School of \\ Medicine, Sapporo, Japan
}

\section{Keywords}

Malignant melanoma - Vemurafenib - Brain metastasis - Drug resistance - BRAF mutation .

Heterogeneous lesion · LDH

\begin{abstract}
Vemurafenib is an inhibitor of the BRAF mutation and has been approved by the Food and Drug Administration as a treatment option for patients with unresectable melanoma without brain metastasis. In the literature, vemurafenib has been reported to be also effective against brain metastasis. We encountered 3 cases with brain metastasis on vemurafenib therapy. In these cases, vemurafenib was clinically effective against metastatic lesions other than those in the brain. The brain lesions developed after the metastatic lesion had occurred. Therefore, we assume that the melanomas of the patients acquired resistance against vemurafenib. The brain metastases were treated with the cyberknife. Patients 1 and 2 without LDH elevation are still alive, but patient 3 with abnormal LDH elevation died despite the treatment. We
\end{abstract}




\section{Case Reports in Oncology}

Case Rep Oncol 2017;10:290-295

DOI: $10.1159 / 000461576$

(C) 2017 The Author(s). Published by S. Karger AG, Base www.karger.com/cro

Imafuku et al.: Sudden Onset of Brain Metastasis despite the Use of Vemurafenib for Another Metastatic Lesion in Malignant Melanoma Patients

need to carefully follow patients on vemurafenib therapy because brain metastasis can suddenly occur even if the metastatic lesion has decreased clinically. The therapeutic effect of vemurafenib against brain metastasis is poor in cases with LDH elevation.

(C) 2017 The Author(s)

Published by S. Karger AG, Basel

\section{Introduction}

Vemurafenib is a BRAF inhibitor for unresectable malignant melanoma. It is known that vemurafenib quickly shrinks metastatic lesions. However, once a tumor is drug resistant, the metastatic lesions quickly become larger again. Brain metastasis is frequently observed in patients with metastatic melanoma [1]. In a phase III trial of vemurafenib, patients with brain metastasis were excluded [2]. Therefore, it is not known whether vemurafenib is truly effective when treating brain metastasis. However, some studies have reported that brain metastasis responds to vemurafenib [3]. Unfortunately, it does not become clear in those reports whether vemurafenib is spontaneously effective against both brain and other metastases. Here, we report 3 cases of brain metastasis with sudden onset despite vemurafenib administration. The patient characteristics are shown in Table 1.

\section{Case Report}

A total of 9 patients with BRAF mutation have been treated with vemurafenib at our institution. Six patients are categorized as stable disease with partial response. In those, vemurafenib was administrated at a dosage of $960 \mathrm{mg}$ twice a day. The other three patients were also categorized as partial response before brain metastasis occurred. In all of them, brain metastasis was suddenly detected on magnetic resonance imaging (MRI).

Patient 1 is a 43-year-old female with lung metastasis when she started vemurafenib therapy. Her lung metastasis reduced gradually (Fig. 1a, b). However, 3 months after starting the vemurafenib therapy, brain metastasis was visible on MRI (LDH was 161, Fig. 1c). The vemurafenib therapy was continued for another 3 months. Cyberknife treatment was performed, but the brain metastasis enlarged gradually (LDH was 244, Fig. 1d).

Patient 2 was a 53-year-old male with lung and liver metastasis when he started vemurafenib therapy. His metastatic site was controlled well. After 6 months on vemurafenib, an MRI revealed brain metastasis (Fig. 1e). The LDH level was $>600$. Therefore, the patient was switched to nivolumab therapy with cyberknife. Unfortunately, the metastatic lesion became rapidly bigger (Fig. 1f). The brain metastasis could not be controlled, and the LDH level promptly increased to 5,665. Despite treatment, the patient eventually died 3 months later due to brain metastasis.

Patient 3 is a 75-year-old male with lung and liver metastasis when he started vemurafenib therapy. After 4 months on vemurafenib, disturbances of consciousness suddenly appeared. MRI scans revealed meningeal seeding (LDH was 240, Fig. 1g), whereas the patient's other metastatic lesions appeared to be reduced on computed tomography. 


\section{Case Reports in Oncology}

Case Rep Oncol 2017;10:290-295

DOI: $10.1159 / 000461576$

(C) 2017 The Author(s). Published by S. Karger AG, Base www.karger.com/cro

Imafuku et al: Sudden Onset of Brain Metastasis despite the Use of Vemurafenib for

Another Metastatic Lesion in Malignant Melanoma Patients

\section{Discussion}

It is known that melanoma cells develop drug resistance within a few months and that the progression of acquired drug resistance induces the amplification of melanoma cells [4]. On the other hand, vemurafenib has a high molecular weight; therefore, it cannot easily cross the blood-brain barrier [5]. In addition, the primary and metastatic melanoma lesions were sometimes heterogeneous [6]. Based on these facts, some oncologists might conclude that our patients developed brain metastasis due to a BRAF mutation or the administered drug's inability to cross the blood-brain barrier. However, we speculate that our patients' tumors acquired drug resistance against vemurafenib since their metastatic lesions kept growing despite the patients being on vemurafenib. There are many reports about drug resistance against vemurafenib. It is well known that acquired drug resistance induces MAPK reactivation based on the activation of secondary mutations in NRAS, MEK, and others [7]. Unfortunately, we could not investigate any other mutations in our patients.

When treating patients with brain metastasis, it is important to monitor LDH fluctuation. Even if a metastatic lesion other than brain metastasis is well controlled, it is possible that the LDH level may gradually increase, and brain metastasis might suddenly occur, as in patients 1 and 3 . When the LDH level increases, it is difficult to control brain metastasis, even with cyberknife treatment. Patient 2 had a high LDH level, and the tumor activity was very aggressive. Therefore, LDH can be a useful biomarker for tumor activity after switching medication. However, even when the LDH level is normal, it is necessary to carefully monitor tumor activity. To our knowledge, no reports of brain metastasis occurring suddenly during vemurafenib treatment have been published. We believe that this report is relevant for patients with malignant melanoma harboring the BRAF mutation and being on vemurafenib therapy.

\section{Statement of Ethics}

The authors have no ethical conflicts to disclose.

\section{Disclosure Statement}

The authors have no conflicts of interest and no funding sources to disclose.

\section{References}

1 Zakrzewski J, Geraghty LN, Rose AE, Christos PJ, Mazumdar M, Polsky D, Shapiro R, Berman R, Darvishian F, Hernando E, Pavlick A, Osman I: Clinical variables and primary tumor characteristics predictive of the development of melanoma brain metastases and post-brain metastases survival. Cancer 2011;117:1711-1720.

7 Chapman PB, Hauschild A, Robert C, et al: Improved survival with vemurafenib in melanoma with BRAF V600E mutation. N Engl J Med 2011;364:2507-2516. 


\section{Case Reports in Oncology}

Imafuku et al:: Sudden Onset of Brain Metastasis despite the Use of Vemurafenib for Another Metastatic Lesion in Malignant Melanoma Patients

Dummer R, Goldinger SM, Turtschi CP, Eggmann NB, Michielin O, Mitchell L, Veronese L, Hilfiker PR, Felderer L, Rinderknecht JD: Vemurafenib in patients with BRAF(V600) mutation-positive melanoma with symptomatic brain metastases: final results of an open-label pilot study. Eur J Cancer 2014;50:611-621.

-4 Shi H, Moriceau G, Kong X, Lee MK, Lee H, Koya RC, Ng C, Chodon T, Scolyer RA, Dahlman KB, Sosman JA, Kefford RF, Long GV, Nelson SF, Ribas A, Lo RS: Melanoma whole-exome sequencing identifies (V600E)B-RAF amplification-mediated acquired B-RAF inhibitor resistance. Nat Commun 2012;3:724. Fonkem E, Uhlmann EJ, Floyd SR, Mahadevan A, Kasper E, Eton O, Wong ET: Melanoma brain metastasis: overview of current management and emerging targeted therapies. Expert Rev Neurother 2012;12:1207-1215.

6 Colombino M, Capone M, Lissia A, Cossu A, Rubino C, De Giorgi V, Massi D, Fonsatti E, Staibano S, Nappi O, Pagani E, Casula M, Manca A, Sini M, Franco R, Botti G, Caraco C, Mozzillo N, Ascierto PA, Palmieri G: BRAF/NRAS mutation frequencies among primary tumors and metastases in patients with melanoma. J Clin Oncol 2012;30:2522-2529.

-7 Trunzer K, Pavlick AC, Schuchter L, et al: Pharmacodynamic effects and mechanisms of resistance to vemurafenib in patients with metastatic melanoma. J Clin Oncol 2013;31:1767-1774. 


\section{Case Reports in Oncology}

Case Rep Oncol 2017;10:290-295

DOI: $10.1159 / 000461576$

(C) 2017 The Author(s). Published by S. Karger AG, Basel www.karger.com/cro

Imafuku et al.: Sudden Onset of Brain Metastasis despite the Use of Vemurafenib for Another Metastatic Lesion in Malignant Melanoma Patients
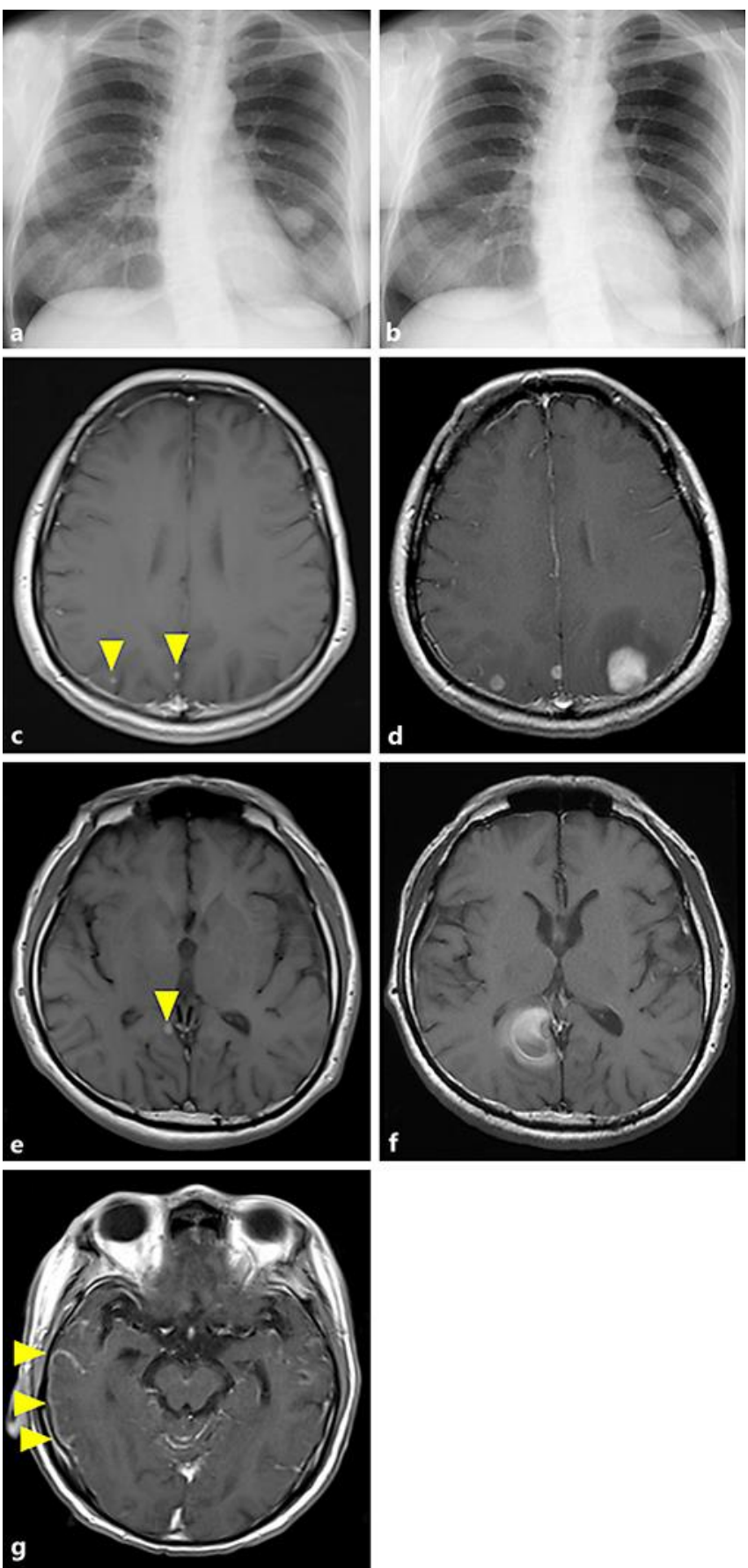

KARGER 
Imafuku et al.: Sudden Onset of Brain Metastasis despite the Use of Vemurafenib for Another Metastatic Lesion in Malignant Melanoma Patients

Fig. 1. Plain X-ray and cerebral MRI (T1-weighted image). a Plain chest X-ray of patient 1 before vemurafenib: a metastatic lesion is detected in her left lung. $\mathbf{b}$ Plain chest X-ray of patient 1 after vemurafenib: the size of the metastatic lesion is reduced to approximately half. c Patient 1: 3 months after starting vemurafenib therapy, several brain metastases are present in the occipital lobe (yellow arrowheads). d Patient 1: 3 months later (i.e. 6 months after starting vemurafenib therapy), the metastatic tumors have grown rapidly despite vemurafenib therapy. e Patient 2: 4 months after starting vemurafenib therapy, several brain metastases are present in the occipital lobe. f Patient 2: 4 months later (i.e. 8 months after starting vemurafenib therapy), the metastatic tumors have grown rapidly. g Patient 3: hyperintensity was detected on the surface of the right temporal lobe.

Table 1. Patient characteristics

\begin{tabular}{llll}
\hline & Patient 1 & Patient 2 & Patient 3 \\
\hline Age, years & 43 & 53 & 75 \\
Sex & Female & Male & Male \\
Weight, kg & 57 & 64 & 56 \\
Place of primary lesion & Left thigh & Left shoulder & Chest \\
Place of metastatic lesion & Lungs & Lungs, liver & Lungs, muscle \\
Initial dose, mg & $960^{\text {a }}$ & $960^{\text {a }}$ & $960^{\text {a }}$ \\
Maintenance dose, mg & $480^{\text {b }}$ & $720^{\text {a }}$ & $720^{\text {a }}$ \\
Adverse events & Rash & Uveitis, arthralgia & Rash \\
LDH at brain metastasis & 161 & 617 & 240 \\
Maximal LDH & 244 & 5,665 & 666 \\
Time to brain metastasis after & & & \\
$\quad$ first vemurafenib administration, months & 3 & 6 & 5 \\
RECIST until brain metastasis & PR & PR & PR \\
Outcome & Alive & Dead & Alive \\
\hline
\end{tabular}

PR, partial response.

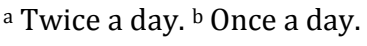

